\title{
Pressure Drop and Heat Transfer Characteristics of a High-temperature Printed Circuit Heat Exchanger
}

Minghui Chen ${ }^{\mathrm{a}}$, Xiaodong Sun ${ }^{\mathrm{a}}$, Richard N. Christensen ${ }^{\mathrm{a}, 1}$, Isaac Skavdahl ${ }^{\mathrm{b}}$, Vivek Utgikar ${ }^{\mathrm{b}}$, Piyush Sabharwall ${ }^{\mathrm{c}}$

\footnotetext{
${ }^{a}$ Nuclear Engineering Program, The Ohio State University, Columbus, OH 43210, USA

${ }^{\mathrm{b}}$ Department of Chemical and Materials Engineering, University of Idaho, Moscow, ID 83844, USA

${ }^{\mathrm{c}}$ Idaho National Laboratory, Idaho Falls, ID 83415, USA

${ }^{1}$ Current address: Nuclear Engineering Program, University of Idaho - Idaho Falls, 995 University Place, Idaho Falls, Idaho 83401, USA
}

\begin{abstract}
Printed circuit heat exchanger (PCHE) is one of the leading intermediate heat exchanger (IHX) candidates to be employed in the very-high-temperature gas-cooled reactors (VHTRs) due to its capability for hightemperature, high-pressure applications. In the current study, a reduced-scale zigzag-channel PCHE was fabricated using Alloy 617 plates for the heat exchanger core and Alloy $800 \mathrm{H}$ pipes for the headers. The pressure drop and heat transfer characteristics of the PCHE were investigated experimentally in a hightemperature helium test facility (HTHF) at The Ohio State University. The PCHE inlet temperatures and pressures were varied up to $464^{\circ} \mathrm{C} / 2.7 \mathrm{MPa}$ for the cold side and $802{ }^{\circ} \mathrm{C} / 2.7 \mathrm{MPa}$ for the hot side, respectively, while the maximum helium mass flow rates on both sides of the PCHE reached $39 \mathrm{~kg} / \mathrm{h}$. The corresponding maximum channel Reynolds number was approximately 3,558, covering the laminar flow and laminar-to-turbulent flow transition regimes. New pressure drop and heat transfer correlations for the current zigzag channels with rounded bends were developed based on the experimental data. Comparisons between the experimental data and the results obtained from the available PCHE and straight circular pipe
\end{abstract}


correlations were conducted. Compared to the heat transfer performance in straight circular pipes, the zigzag channels provided little advantage in the laminar flow regime but significant advantage near the transition flow regime.

\section{Introduction}

Advanced nuclear reactors, such as the very-high-temperature gas-cooled reactors (VHTRs) from the Generation IV nuclear systems, are capable of generating electricity with high efficiencies and providing high-temperature industrial process heat. VHTRs are designed with the capability of delivering highpressure, high-temperature helium to a power conversion unit (PCU) for electricity production and an industrial plant for process heat applications. The helium temperature at the reactor core outlet is designed to be $750 \sim 850^{\circ} \mathrm{C}$ during the first development phase and is expected to be increased in the later development [1]. With such high temperatures, VHTRs offer a wide range of applications. The electric power production may use a Rankine cycle with high-pressure steam generators, or a direct Brayton cycle with gas turbines using the primary helium coolant as the working fluid, or an indirect Brayton cycle using a secondary fluid, such as helium or supercritical carbon dioxide $\left(\mathrm{s}-\mathrm{CO}_{2}\right)$ [2]. The process heat applications may include hydrogen production, petroleum refining, bio-fuels production, and production of chemical feed stocks for use in the fertilizer and chemical industries [3]. The efficiency of the electricity production and process heat applications of VHTRs is critically dependent upon intermediate heat exchangers (IHXs), which are a key component in transferring the thermal energy from the primary coolant to the secondary coolant.

The IHX serves to isolate the reactor primary system from electricity generation and process heat application plants, and therefore must be robust enough to maintain the entire system integrity during normal and off-normal conditions. Since helium typically has a low heat transfer capability due to its low volumetric thermal capacity and low thermal conductivity, a compact heat exchanger with a high surface 
area to volume ratio (generally, higher than $700 \mathrm{~m}^{2} / \mathrm{m}^{3}$ [4]) is preferable to be employed as an IHX in the VHTRs. A printed circuit heat exchanger (PCHE) stands out from several heat exchanger candidates due to its high effectiveness, compactness, robustness, and its ability to withstand high pressures and temperatures $[3,5]$. PCHEs are plate-type compact heat exchangers in which flow channels (typically, channels with a small hydraulic diameter) are etched into flat metal plates using a photochemical machining process. There are several PCHE designs with respect to the flow channel geometries, such as straight, zigzag, wavy, S-shape finned, and airfoil finned channels. The etched metal plates are then grinded and lapped to remove scratches on the plate surfaces followed by making the etched plates flat and parallel. Finally, the plates are stacked together with a prescribed arrangement configuration and diffusion bonded to create a high-integrity solid block before flow distribution headers are attached to the heat exchanger block.

Over the past decade, a number of studies related to PCHEs have been conducted in U.K., Japan, South Korea, and U.S. Nikitin et al. [6] investigated heat transfer and pressure drop characteristics of a 3-kW PCHE with compactness of $1050 \mathrm{~m}^{2} / \mathrm{m}^{3}$ in a supercritical $\mathrm{CO}_{2}$ loop. Tsuzuki et al. [7] conducted threedimensional thermal-hydraulic simulations of a PCHE with S-shape finned channels and concluded that S-shape finned channels have the same thermal performance as zigzag channels while decrease pressure drop to one-fifth of that in conventional zigzag-channel PCHE. In addition, these authors found that there are flow reversal occurred in the zigzag channels. Ngo et al. [8] experimentally investigated the thermalhydraulic characteristics of PCHEs with zigzag and S-shape finned channels. Both PCHEs have higher heat transfer performance than the conventional shell-and-tube heat exchangers. The PCHE with zigzag channels has a 24-34\% higher Nusselt number than the PCHE with S-shape finned channels. However, the pressure drop in the zigzag channels is 4-5 times larger than that in the S-shape finned channels for the same Reynolds numbers. Kim [9] conducted comprehensive numerical and experimental studies on zigzag-channel PCHEs based on helium-helium, helium-water, and gas mixture (helium and $\mathrm{CO}_{2}$ )-water working fluid combinations. Correlations for calculating the Fanning friction factor and Nusselt number 
were proposed for zigzag-channel PCHEs based on the channel angle, pitch length, and hydraulic diameter. Mylavarapu et al. [10] designed and constructed a high-temperature helium test facility (HTHF) that can facilitate compact heat exchanger thermal-hydraulic testing using helium as the working fluid at temperatures and pressures up to $850^{\circ} \mathrm{C}$ and $3 \mathrm{MPa}$ with helium mass flow rates ranging from 15 to 49 kg/h. Two similar straight-channel PCHEs were fabricated using Alloy 617 plates for the heat exchanger core and were tested in the HTHF in a counter flow configuration for their thermal-hydraulic performance. The authors proposed correlations to determine the hydrodynamic entrance length and the Fanning friction factor in the hydrodynamic entrance region for laminar flow in the semicircular duct [10, 11]. Bartel et al. [12] performed a comparative study of PCHE designs for a typical VHTR and concluded that the zigzag-channel PCHE with a pitch angle of $15^{\circ}$ provided the best performance in heat transfer while incurring a moderate pressure drop penalty as compared to the straight-channel PCHE. Chen et al. [13] presented a detailed fabrication procedure of a counter-flow PCHE with zigzag channels that can be used in high-temperature, high-pressure conditions and performed a preliminary thermal-hydraulic investigation of the fabricated PCHE. Khan et al. [14] performed three-dimensional steady-state conjugate heat transfer simulations to study zigzag-channel PCHEs with various incline angles and presented detailed thermal-hydraulic characteristics of the PCHE. It was found that there are flow enhancement and secondary flow regimes in zigzag channels. Flow enhancement increases heat transfer while the secondary flow due to flow separation and vortex formation would affect heat transfer augmentation. In addition, the authors demonstrated better thermal-hydraulic performance for wavy-channel PCHEs as compared to straight channel based PCHEs.

Although many studies have been performed on PCHEs, few experiments have been conducted on the thermal-hydraulic performance of zigzag-channel PCHEs under VHTRs’ typical operating conditions with temperatures near $750^{\circ} \mathrm{C}$ or above. In addition, most of studies in the literature used acquired PCHEs from a commercial vendor and there could be relatively large uncertainties in the geometrical parameters of the heat exchangers, which would propagate to the uncertainties associated with the correlations 
developed based on those experimental data. The aim of the current research is therefore to improve our understanding of the thermal-hydraulic performance of zigzag-channel PCHEs under high-temperature conditions. In this paper, a reduced-scale counter-flow PCHE with zigzag channels was designed, fabricated, and then tested for its thermal-hydraulic performance in the HTHF at temperatures and pressures up to $802^{\circ} \mathrm{C}$ and $2.7 \mathrm{MPa}$, respectively. The experimental data were compared with the available correlations developed for PCHEs. New pressure drop and heat transfer correlations based on the experimental data were suggested for the current zigzag-channel PCHE.

\section{Printed Circuit Heat Exchanger and Experimental Facility}

\subsection{Printed circuit heat exchanger}

A reduced-scale zigzag-channel PCHE was fabricated using 1.6-mm thick Alloy 617 plates for the heat exchanger core and Alloy $800 \mathrm{H}$ pipes for its headers. A total of eight hot and eight cold plates with 11 zigzag channels in each of the plates were first stacked in an alternating manner and then diffusion bonded together to form a metal block, i.e., the heat exchanger core. Figure 1 shows one hot-side plate and one cold-side plate. The cross section of the fluid passages is approximately semicircular with a nominal diameter of about $2 \mathrm{~mm}$ and a pitch of $2.5 \mathrm{~mm}$ in the span-wise direction. The shape of the flow passage is zigzag with a curvature (radius of curvature is $4 \mathrm{~mm}$ ) at the tip of each bend. The angle between the flow direction in the zigzag channel and the edge of the block, i.e., the flow direction along the heat exchanger length, is $15^{\circ}$. The PCHE was designed in such a way that each side can withstand the maximum pressure of $3 \mathrm{MPa}$ in the HTHF. The flow passages in each plate of the PCHE were made by applying a photochemical etching technique, which used strong chemical etchants to remove the selected area on the surface of the plates. Four 20.7-mm diameter through-holes and four 6.35-mm diameter small through-holes were also made on each of the total 16 plates during the chemical etching process. The four larger holes were used as plena to direct the flow into and out of the PCHE flow channels. The other four smaller holes on the plates were used for alignment during the diffusion bonding process. Four pins were 
inserted into those four smaller holes before diffusion bonding to prevent the plates from sliding when adding large loads onto the PCHE block. Two 12.7-mm thick plates, one at the top of the heat exchanger block and the other at the bottom, were also applied to provide an additional strength to the PCHE block. In addition, the topmost plate provided a strong base for joining the four headers. The detailed fabrication procedures are referred to References 13 and 15. A photo of the actual PCHE assembly with four headers attached is shown in Fig. 2. The dimensions of the PCHE block are $339.1 \mathrm{~mm}$ in length, $126 \mathrm{~mm}$ in width, and $50.8 \mathrm{~mm}$ in height. The headers were made from one-inch nominal pipe size (NPS) Alloy $800 \mathrm{H}$ pipes since the piping size in the test facility is one inch NPS (corresponding to a pipe schedule of 160).

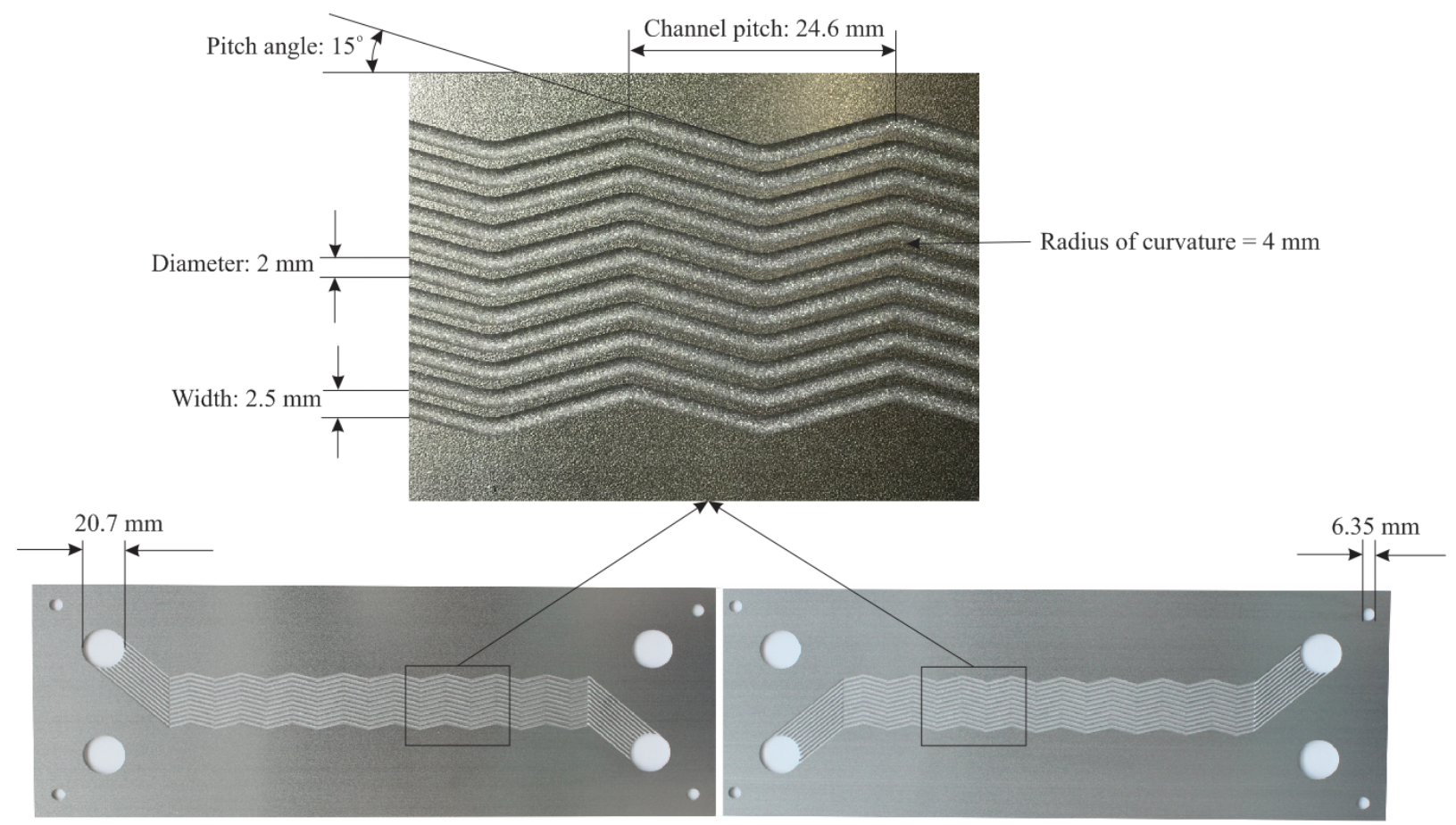

Fig. 1. Images of one hot-side plate and one cold-side plate. 


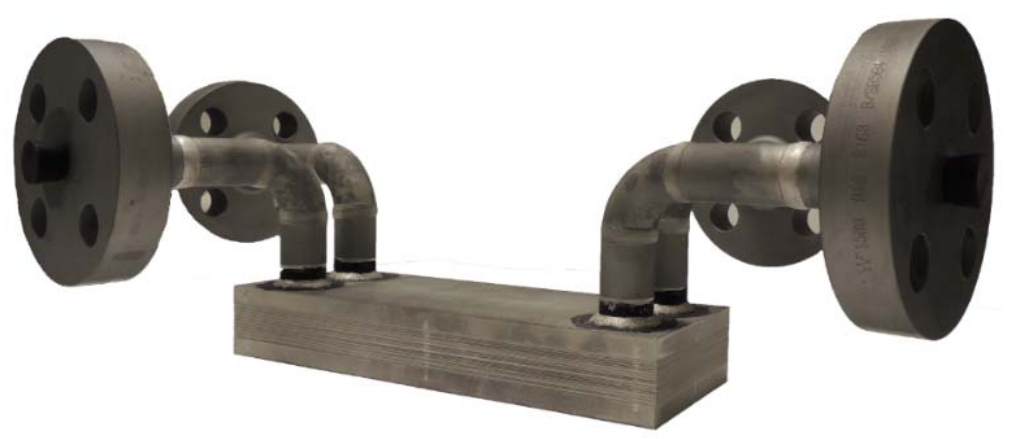

Fig. 2. Photo of the zigzag-channel PCHE with four headers assembled.

\subsection{High-temperature helium test facility}

Experiments were performed to examine the thermal-hydraulic characteristics of the zigzag-channel PCHE under various steady-state conditions. The experimental study capitalized on the HTHF at The Ohio State University. Figure 3 is a simplified layout of the HTHF showing the helium flow path for heat exchanger testing, where one of the two previously-fabricated straight-channel PCHEs $[3,10]$ was replaced by the current reduced-scale zigzag-channel PCHE (i.e., the PCHE being tested). The HTHF was designed and constructed to facilitate thermal-hydraulic performance testing of compact heat exchangers at temperatures and pressures up to $850^{\circ} \mathrm{C}$ and $3 \mathrm{MPa}$, respectively $[3,10]$. The HTHF consists of a preheater and a main-heater to heat helium to elevated temperatures, a gas booster to boost the helium pressure to overcome the pressure drop in the loop, a cooler to cool helium to low temperatures before returning to the gas booster, piping, high-temperature valves, and various instruments. The pre-heater and main-heater are similar electric heaters with the same maximum heating capacity of $23 \mathrm{~kW}$. Three heating elements, each having a maximum capacity of approximate $7.6 \mathrm{~kW}$, were embedded on the inner surface of the ceramic fiber insulation and were virtually free-radiating. The maximum heating element temperature can reach $1,300{ }^{\circ} \mathrm{C}$, enabling high-temperature tests in the test loop. The helium flow in the test facility had periodical fluctuations due to the reciprocating actions of the booster, which is a singlestage double-acting air-drive booster. Therefore, an inline air-drive pressure regulator valve, in addition to a helium surge tank, was installed at a downstream location of the booster to damp the flow oscillations. 
To assure the experimental data quality, all the instruments were calibrated against standards traceable to the National Institute of Standards and Technology (NIST).

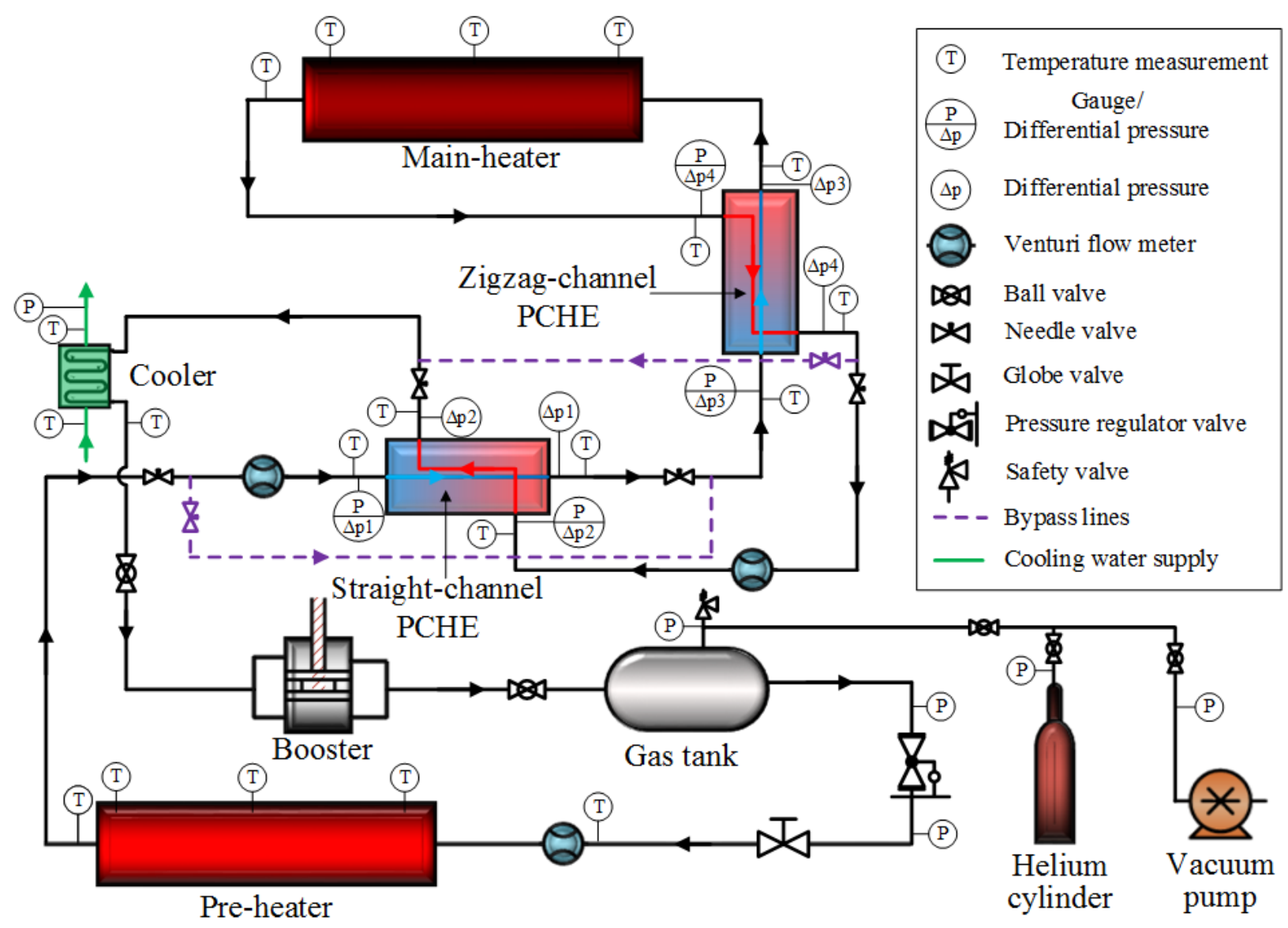

Fig. 3. Layout of the high-temperature helium test facility.

\section{Experimental Data Reduction Method}

\subsection{Fanning friction factor}

The test PCHE was installed in the HTHF for the determination of its pressure drop and heat transfer characteristics. The first step was to determine the friction characteristics, i.e., Fanning friction factor. The approach described by Chen [15] was adopted for this task. Figure 4 displays the pressure drop 
measurement locations on one side of the PCHE. Two differential pressure transducers were installed on each side of the test PCHE to measure pressure drops across both the hot and cold sides of the PCHE.

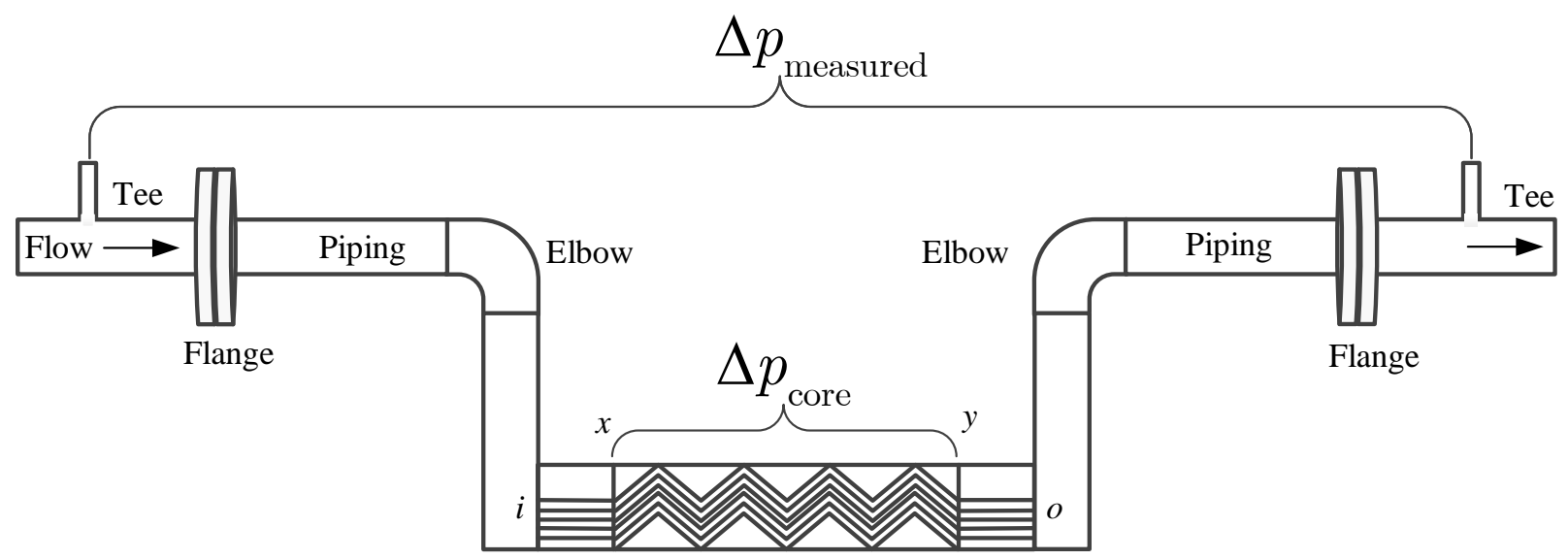

Fig. 4. PCHE set-up schematic and illustration of pressure drop contributions.

It can be seen from Fig. 4 that the total pressure drop measured on each side includes several contributors: pressure loss associated with the heat exchanger core; pressure loss associated due to fluid distribution devices, such as the distribution headers, plena; additional pressure loss at the straight-channel regions (i.e., regions between surfaces $i$ and $x$, and $y$ and $o$ as shown in Fig. 4) at the inlet and outlet of the exchanger (the average flow lengths for $i-x, x-y$, and $y$-o are $39.94 \mathrm{~mm}, 203.3 \mathrm{~mm}$, and $34.11 \mathrm{~mm}$, respectively); and pressure loss associated with the piping, fittings (tees and elbows), and flanges between the differential pressure measurement taps. Therefore, factors that cause pressure drop in the current PCHE are: (1) flow frictional losses in the heat exchanger core; (2) flow momentum rate change; (3) pressure drop associated with sudden contraction at the channel inlets; (4) pressure change associated with sudden expansion at the channel exits; and (5) pressure drops in straight-channel inlet and outlet regions, piping, fittings, and flanges.

To determine the friction characteristics of the PCHE core, it is necessary to exclude the pressure drop contributions that are not associated with the heat exchanger core friction from the experimentally- 
measured pressure drop values. Other pressure drop contributions not related to the heat exchanger core pressure drop were subtracted with the help of available empirical correlations in the literature. The experimental Fanning friction factor was then estimated from the core pressure drop for the zigzag channels of the PCHE. For the heat exchanger core frictional characteristics, the hot-side and cold-side pressure drops were separately obtained under elevated-temperature test conditions. Some assumptions made for pressure drop analysis were as follows: (1) the incoming flow is steady; (2) the fluid is uniformly distributed into each of the channel from the headers; (3) the channel geometry is identical for all channels on each of the cold and hot sides; (4) gravity effect is neglected since the heat exchanger is horizontally oriented; and (5) the cross sections of the flow channel passages are exactly semicircular. The total measured pressure drop is the sum of all the pressure drop contributions and can be expressed as

$$
\begin{aligned}
\Delta p_{\text {measured }}= & \left(G^{2} / 2 \rho_{i}\right)\left[\left(1-\sigma^{2}+K_{c}\right)-\left(1-\sigma^{2}-K_{e}\right)\left(\rho_{i} / \rho_{o}\right)\right]+ \\
& \left(G^{2} / 2 \rho_{i}\right)\left[2\left(\rho_{i} / \rho_{o}-1\right)+4 f\left(l / d_{h}\right) \rho_{i}\left(1 / \rho_{m}\right)\right]+ \\
& \left(\Delta p_{\text {straight-channel regions }}+\Delta p_{\text {fittings }}+\Delta p_{\text {piping }}+\Delta p_{\text {flanges }}\right) .
\end{aligned}
$$

The variables can be referred from the Nomenclature Section. The Fanning friction factor, $f$, the only unknown in Eq. (1), can be determined from the experimental data. For flow in a straight circular pipe, Eq. (2) can be used to calculate the Fanning friction factor for fully-developed flow.

$$
f=\left\{\begin{array}{l}
\frac{16}{\operatorname{Re}}, \quad \operatorname{Re} \leq 2,300 \\
\frac{1}{4}\left[\frac{1}{(0.79 \ln \operatorname{Re}-1.64)}\right]^{2}, \quad \operatorname{Re}>2,300
\end{array}\right.
$$

\subsection{Heat transfer coefficient}

In the present experimental study, no local internal fluid and wall temperature measurements were available. Therefore, local and average convective heat transfer coefficients on both the hot and cold sides 
of the heat exchanger cannot be obtained directly. A typical method used for determining the average heat transfer coefficient on either side of the PCHE is described in this section.

From the PCHE fabrication aspects, the hot-side heat transfer area and cold-side heat transfer area are the same (i.e., $A_{s, h}=A_{s, c}=A_{s}$ ). The overall thermal resistance is given by:

$$
\frac{1}{U A_{s}}=\frac{1}{h_{h} A_{s, h}}+\frac{1}{h_{c} A_{s, c}}+R_{w} .
$$

For a determined fluid, (i.e., helium), the heat transfer model is only a function of the Reynolds number $[6,9]$. The Prandtl number of helium was treated as a constant since its variations over the testing temperature range in the current study were less than $1 \%$. The heat transfer coefficient for either side can be calculated from the Nusselt number, expressed as:

$$
\mathrm{Nu}=c \operatorname{Re}^{a}
$$

where $a$ and $c$ are constants. Therefore, the average heat transfer coefficient $h$ is given by:

$$
h=\frac{\mathrm{Nu} \lambda}{d_{h}}=\frac{c \operatorname{Re}^{a} \lambda}{d_{h}}
$$

Substituting Eq. (5) into Eq. (3) yields:

$$
\frac{1}{U d_{h}}=\frac{1}{c \operatorname{Re}_{h}^{a} \lambda_{h}}+\frac{1}{c \operatorname{Re}_{c}^{a} \lambda_{c}}+\frac{R_{w} A_{s}}{d_{h}}
$$


Two unknown constants $a$ and $c$ can be solved by using a nonlinear regression method to minimize the residual $S$ as:

$$
S=\sum_{j=1}^{N}\left[\frac{1}{U_{j} d_{h}}-\frac{R_{w, j} A_{s}}{d_{h}}-\frac{1}{c \operatorname{Re}_{h, j}^{a} \lambda_{h, j}}-\frac{1}{c \operatorname{Re}_{c, j}^{a} \lambda_{c, j}}\right]^{2}
$$

Once $a$ and $c$ are determined, the Nusselt number correlation, i.e., Eq.(4) is confirmed. The heat transfer coefficients for both the hot and cold sides can be calculated separately from the Nusselt number correlation.

To obtain the overall heat transfer coefficient of the zigzag-channel PCHE, it is necessary to determine the effective heat transfer area and consider the heat transfer contributions inside the heat exchanger. Figure 5 is a schematic showing the effective heat transfer area and heat conduction between the two adjacent ports. The heat transferred from the hot side to cold side consists of several contributions: (1) heat conduction from port \#1 to port \#2 and from port \#3 to port \#4; (2) heat transfer in the crossflow configurations in the $\mathrm{X}$ and $\mathrm{Y}$ regions; (3) heat transfer in the countercurrent flow region Z; and (4) heat loss to the surroundings. Four thermocouples were installed at locations 1, 2, 3, and 4 (see Fig. 5) in the HTHF to measure the helium inlet and outlet temperatures of the test PCHE.

Three experimental data points were used to examine the heat conduction from port \#1 to port \#2 and from port \#3 to port \#4, i.e., contribution (1) as mentioned above. The average fraction of the total heat conduction to the total heat transferred from the hot side to cold side was about $0.4 \%$, whereas the overall heat transfer coefficient was $1.1 \%$ higher than that without considering the heat conduction. Changes in the four terminal temperatures resulted in a decrease of the log-mean temperature difference (LMTD) by approximately 1.1\%. A smaller LMTD gave a larger overall heat transfer coefficient when the heat 
conduction between the ports was neglected. In the current study, the heat conduction was considered. Note that the sum of the heat transfer area of regions X, Y, and Z, showing in Fig. 5, is the effective heat transfer area that was used when processing the experimental data.

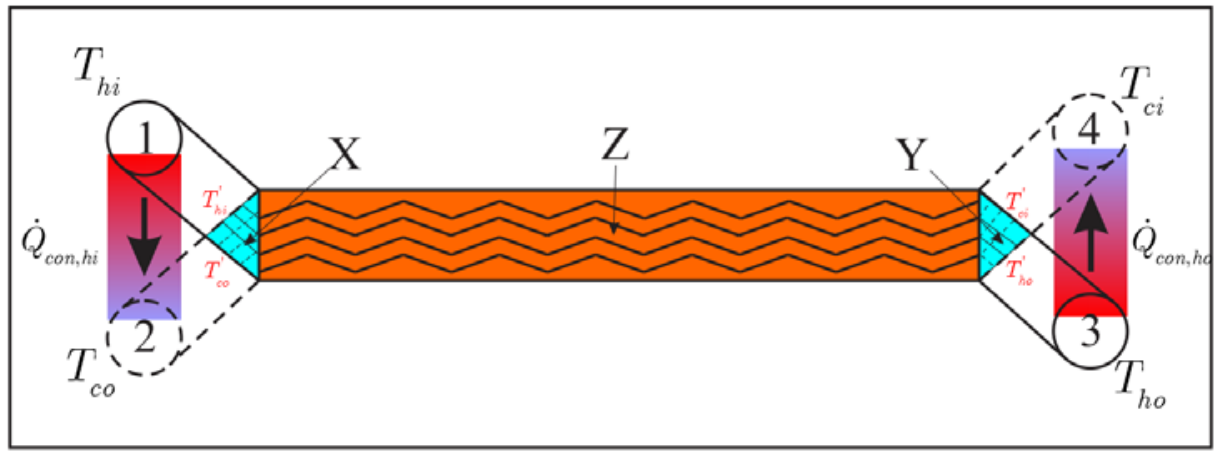

Fig. 5. Schematic of the effective heat transfer area and heat conduction path (regions numbered 1, 2, 3, and 4 represent the hot-side and cold-side inlet/exit ports or plena).

\subsection{Uncertainty analysis}

An uncertainty analysis, considering error propagation, was performed using the root-sum-square method. The uncertainty in the Fanning friction factor was analyzed based on the simplified form of the heat exchanger core friction pressure drop given by

$$
f=\frac{\Delta p}{2} \frac{d_{h}}{l} \frac{\rho A_{c}^{2}}{\dot{m}^{2}}
$$

The uncertainty in Fanning friction factor can be expressed as

$$
\frac{\delta f}{f}=\sqrt{\left(\frac{\delta \Delta p}{\Delta p}\right)^{2}+\left(\frac{\delta d_{h}}{d_{h}}\right)^{2}+\left(2 \frac{\delta \dot{m}}{\dot{m}}\right)^{2}+\left(2 \frac{\delta A_{c}}{A_{c}}\right)^{2}+\left(\frac{\delta l}{l}\right)^{2}+\left(\frac{\delta \rho}{\rho}\right)^{2}} .
$$


The heat transfer model can be obtained by using the nonlinear regression method. A perturbation method was adapted to determine the uncertainties in the obtained heat transfer correlation [16], i.e., uncertainties in constants $a$ and $c$ in Eq. (4). The uncertainties in $\operatorname{Re}_{h}, \operatorname{Re}_{c}, \lambda_{h}, \lambda_{c}, A_{c}, d_{h}, U$, and $R_{w}$ in Eq. (7) can be determined from the experimental data. One of the eight variables was changed to its upper bound and the corresponding values of $a_{1}$ and $c_{1}$ were determined running the nonlinear regression calculation. These two values were compared with the original values (i.e., $a$ and $c$ values) to determine the differences of $\Delta a_{1}$ and $\Delta c_{1}$. The same procedure was repeated for the low bound value of the same variable to obtain $\Delta a_{2}$ and $\Delta c_{2}$. These steps were repeated for the remaining seven variables. There were total 16 regression runs required to compute all individual relative errors in constants $a$ and $c$. Finally, the uncertainties in $a$ and $c$ were calculated separately by taking the root-mean-square method as:

$$
\left\{\begin{array}{l}
\delta a=\sqrt{\frac{1}{n} \sum_{i=1}^{n}\left(\Delta a_{i}^{2}\right)} \\
\delta c=\sqrt{\frac{1}{n} \sum_{i=1}^{n}\left(\Delta c_{i}^{2}\right)}
\end{array}\right.
$$

\section{Experimental Results and Analysis}

The thermal-hydraulic performance testing of the zigzag-channel PCHE was carried out in this section. The test loop was initially vacuumed to the desired vacuum pressure of - 14 psig by using a vacuum pump followed by charging the test loop with helium (with a purity rating of 99.9999\%) to a desired pressure from a helium gas cylinder. The cooler was turned on during all the tests to lower the temperature of helium. The primary measurements taken on the test PCHE consist of the inlet and outlet temperatures, pressure drops across the hot side and cold side, helium mass flow rates on both sides, and gage pressures at the inlet section of both sides. Due to the large thermal inertia of the test facility, it usually took more 
than five hours for the loop to reach the first steady-state condition starting from a room temperature. The criteria of reaching steady-state conditions were based on the standard deviations of the helium temperature, pressure, and helium mass flow rate measurements. The PCHE operation condition was considered as a steady state when standard deviations of the four measured inlet/outlet temperatures were less than $0.5^{\circ} \mathrm{C}$; the standard deviations of the measured static pressure and differential pressure were less than $3 \%$; and the standard deviations of the measured helium mass flow rates were less than $2 \%$ in a twominute duration at a sampling rate of $8 \mathrm{~Hz}[10]$.

Figure 6 shows a total number of 82 experimental data points plotted as functions of the helium mass flow rates, system pressures, and the PCHE hot-side inlet temperatures. The helium mass flow rates were varied from 22 to $39 \mathrm{~kg} / \mathrm{h}$, the system pressures were from 1.6 to $2.7 \mathrm{MPa}$ while the hot-side inlet temperatures were increased from 199 to $802^{\circ} \mathrm{C}$. The maximum helium temperature, located at the exit of the main heater in the test loop, was about $840^{\circ} \mathrm{C}$. The heat exchanger thermal duty for each steady state was calculated based on the average thermal duty on both the hot and the maximum heat exchanger thermal duty was about $15 \mathrm{~kW}$. The heat loss ratio is defined as ratio of the thermal duty difference between the hot side and cold side of the PCHE to the average thermal duty. The heat loss ratios were less than $10 \%$ for all experimental data points.

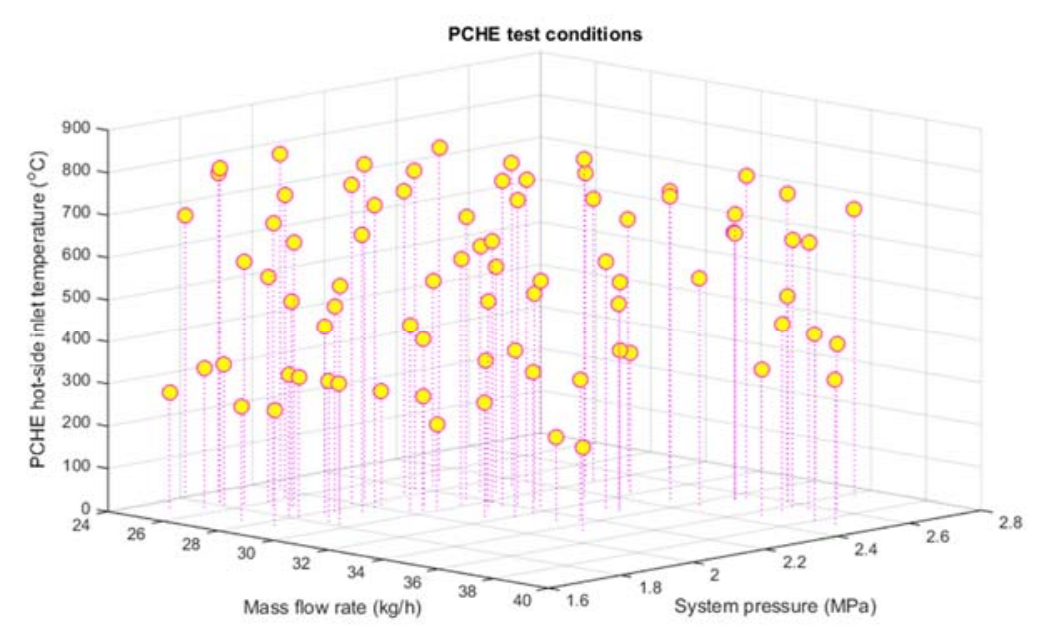

Fig. 6. PCHE test conditions. 


\subsection{Fanning friction factor}

The experimental Fanning friction factors for both the hot and cold sides were obtained using the method described in Section 3.1 and plotted in Figs. 7 and 8 with the uncertainty bars representing the calculated uncertainties described in Section 3.3. In the current experiments, the relative uncertainties in the Reynolds numbers varied from 3.7 to $7.2 \%$. It was observed from these figures that the Fanning friction factors tended to be a weak function of the Reynolds numbers when the Reynolds numbers were greater than about 2,200, which indicated the onset of the laminar-to-turbulent flow transition regime. The uncertainties of all the current experimental data were within $\pm 10 \%$ when the flow variations were less than $2 \%$. In addition, the uncertainties of the friction factors were less than $\pm 6 \%$ and $\pm 10 \%$ for the laminar and transition flow regimes, respectively, at 95\% confidence level.

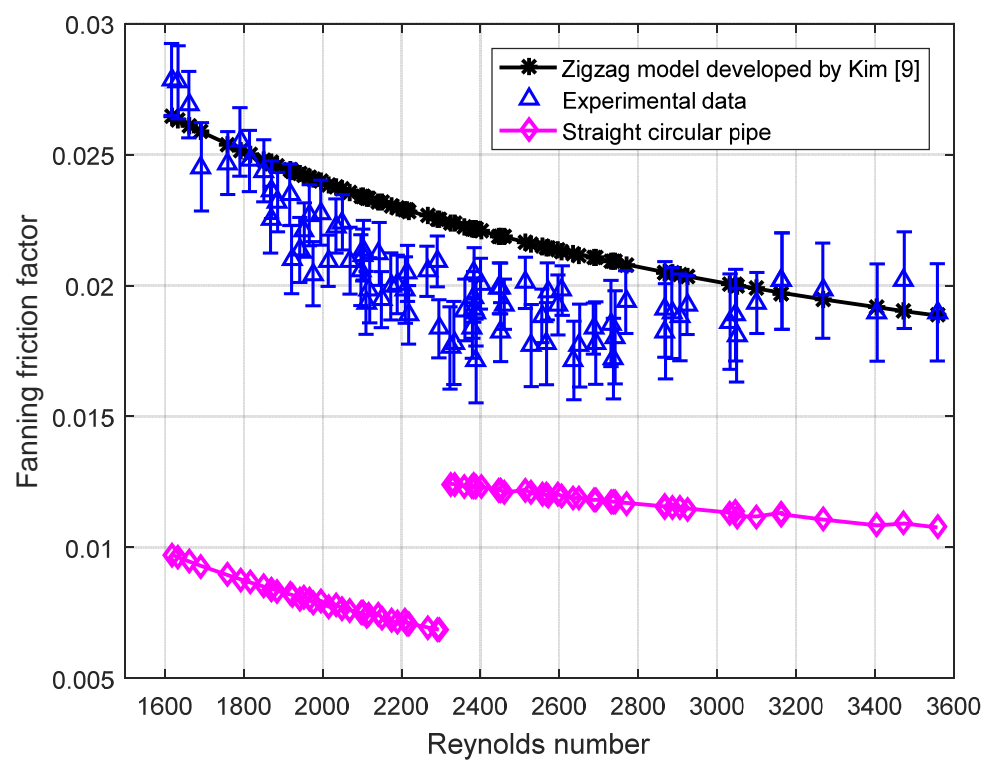

Fig. 7. Cold-side Fanning friction factor. 


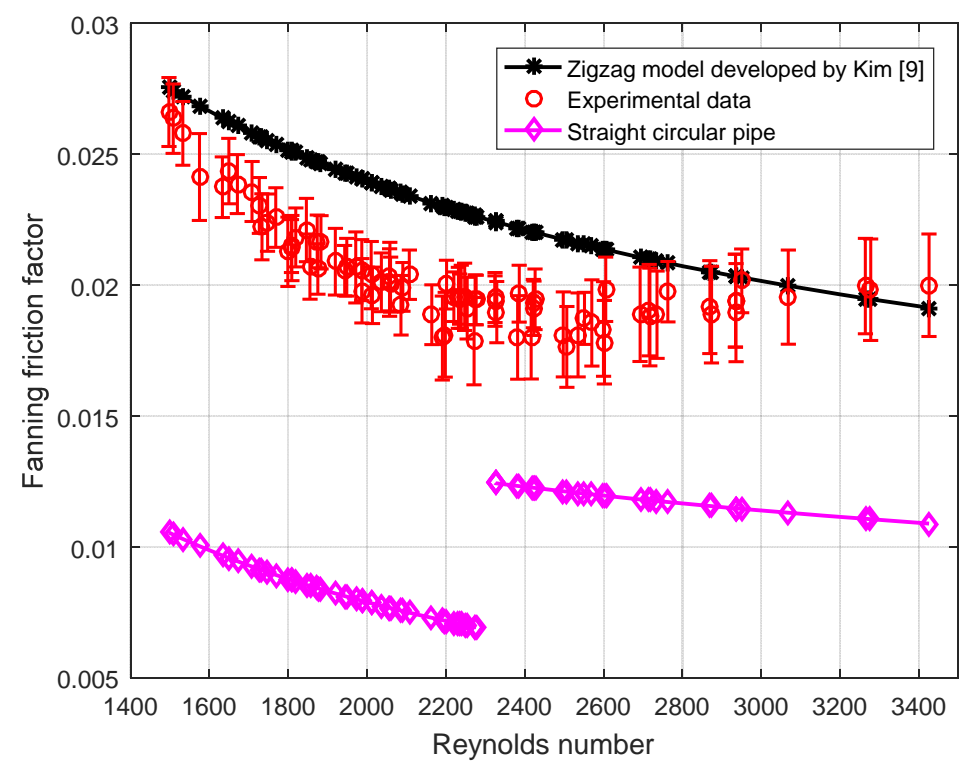

Fig. 8. Hot-side Fanning friction factor.

The experimentally obtained Fanning friction factors were compared with the correlation developed by Kim [9], as also shown in Figs. 7 and 8. The correlation shown in Eq. (2) for fully-developed flow in straight circular pipe are also plotted in these two figures. The results calculated from the correlation developed by Kim slightly over predicted, in most cases, the experimentally obtained Fanning friction factor values. The largest differences were $29 \%$ for both the hot and cold sides. The discrepancies can be attributed to the geometric differences of the flow channels. The zigzag channels used in Kim's computational fluid dynamics simulations were with sharp turns at all bends in the channels, whereas a roundness was induced at each bend in all channels of the currently-tested PCHE. Introducing a roundness to each bend smooths the fluid flow direction change and hence reduces the pressure drop. The second possible reason is due to the difference in the cross section of the flow channels. The channel cross sections in the test PCHE were not exactly semicircular due to the chemical etching properties, while Kim's numerical model was for the zigzag channel with a perfect semicircular cross section. When comparing the experimental data to the results obtained from the straight circular pipe correlations, it was 
found that the zigzag-channel Fanning friction factors were two to three times those in the laminar flow regime and one to two times those in the transition flow regime.

Next, the obtained Fanning friction factors on both the hot and cold sides are plotted in Fig. 9. It was observed that the Fanning friction factors on the hot side and cold side were different, especially in the laminar flow regime, which is postulated to be mainly due to the flow channel geometry. The channels' geometric parameters on the hot side and cold side are not exactly identical due to manufacturing imperfections and tolerance. Fanning friction factors on the cold side were slightly larger than those on the hot side in the laminar flow regime, while in the transition flow regime, they became very similar.

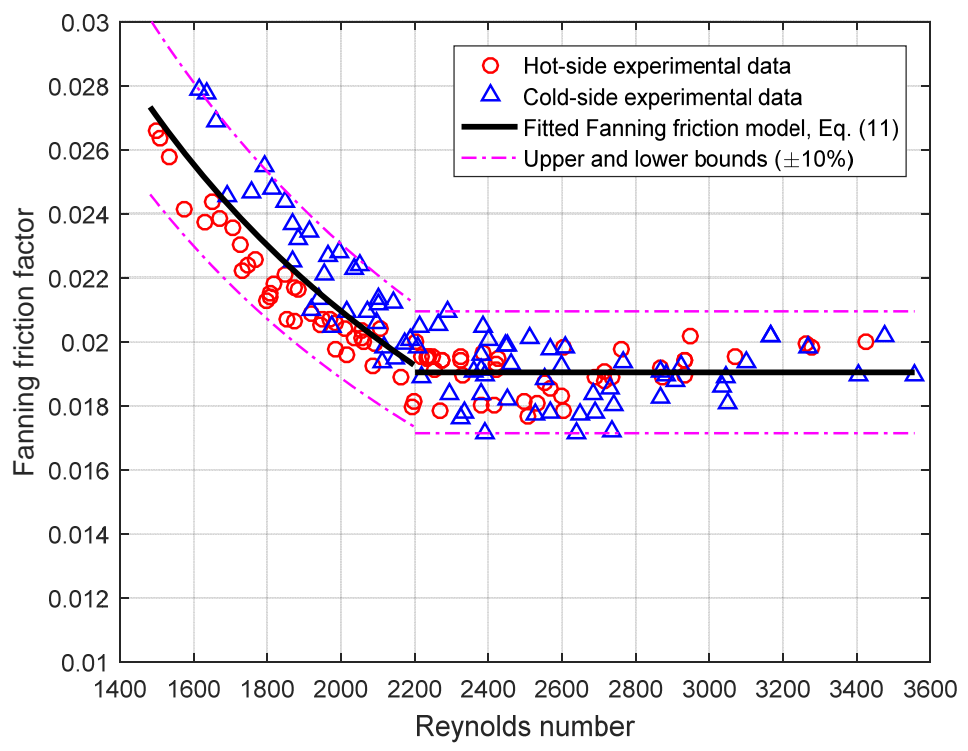

Fig. 9. Experimentally determined Fanning friction factors for both the hot and cold sides and the fitted model.

A power function of the Reynolds number only was utilized to best fit 72 data points in the laminar flow regime for both the hot and cold sides. Since the Fanning friction factors were almost independent of the Reynolds numbers cover the range covered in the transition flow regime, an average Fanning friction factor for the rest 92 experimental data points with twice the standard deviation of 0.000846 , 
corresponding to the 95\% confidence level, was selected for the transition flow regime. Following this exercise, we propose a new Fanning friction correlation for the current zigzag flow channels:

$$
f= \begin{cases}\frac{17.639}{\operatorname{Re}^{0.8861 \pm 0.0017}} & 1,400 \leq \mathrm{Re} \leq 2,200 \\ 0.019044 \pm 0.001692 & 2,200<\operatorname{Re} \leq 3,558\end{cases}
$$

The correlations shown above correlated 98 percent of the 164 experimental data points within \pm 10 percent deviations, as shown in Fig. 9. Note that the proposed correlations are only valid for flow channels of similar geometry studied and the above mentioned Reynolds number ranges.

\subsection{Nusselt number}

Local heat transfer coefficients could not be computed from out experiments since no internal wall and fluid temperature measurements were conducted in the current experimental tests. Therefore, the laminarto-turbulent flow transition behavior could not be identified directly from the experimental data for heat transfer characteristics. The method used to determine the onset of the heat transfer transition regime in the current study was to examine the uncertainties in the constants of the fitted correlations using the nonlinear regression and uncertainty determination methods illustrated in Sections 3.2 and 3.3. Running nonlinear regression fittings for every 15 data points from low to high Reynolds numbers with a step of five data points, the uncertainty of the model in each run can be obtained. Also, the overall uncertainty of the model for the total 82 experimental data points can be calculated. Assuming the 15 data points for each nonlinear regression run were in the laminar regime (the highest Reynolds number is the onset of the transition regime), the uncertainties for all the runs and the overall uncertainty were compared as shown in Fig. 10. It was observed that there was a steep jump and the fitted model uncertainty was larger than the overall uncertainty when Reynolds numbers were around 2,200. The computed uncertainties in the 
transition regime were larger than those in the laminar regime because both the flow and heat transfer behavior in the transition flow regime are chaotic and difficult to predict. It was therefore reasonable to assume the onset of the laminar-to-turbulent heat transfer transition regime occurred at a Reynolds number of approximately 2,200, which was the same as the onset of the transition regime for the fluid flow.

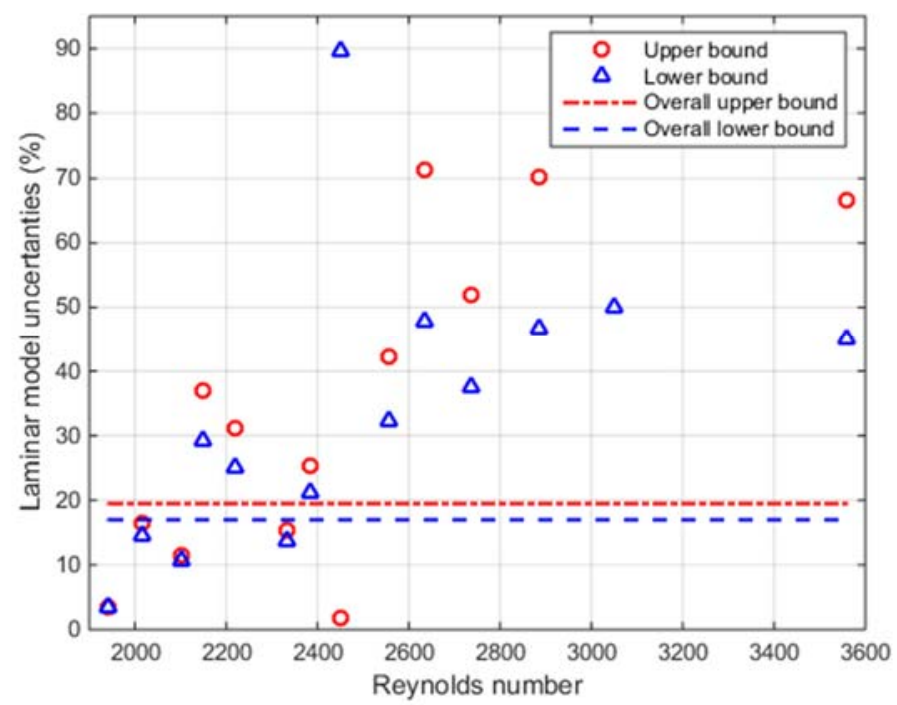

Fig. 10. Fitted model uncertainties.

Following the heat transfer data reduction method illustrated in Section 3.2 for both the laminar and transition flow regimes, fitted correlations can be obtained from 67 data points that were randomly selected from the total 82 experimental data points using the nonlinear regression method as

$$
\mathrm{Nu}=\left\{\begin{array}{ll}
(0.05516 \pm 0.00160) \operatorname{Re}^{(0.69195 \pm 0.00559)} & 1,400 \leq \operatorname{Re} \leq 2,200 \\
(0.09221 \pm 0.01397) \operatorname{Re}^{(0.62507 \pm 0.01949)} & 2,200<\operatorname{Re} \leq 3,558
\end{array} .\right.
$$


The other 15 data points were used to validate the obtained correlations, i.e., Eq. (12) described as follows. A comparison of the overall heat transfer coefficients obtained from the experimental data and those calculated using Eqs. (6) and (12) is presented in Fig. 11, which indicated that the two were in good agreement with each other. The differences were within 3\% for both the laminar and transition regimes. Pra et al. [17] conducted experiments on a Heatric PCHE mock up with similar geometry as the current tested PCHE at lower temperatures and found that Reynolds number power values are from 0.6 to 0.8 , giving similar results as in the present tests.

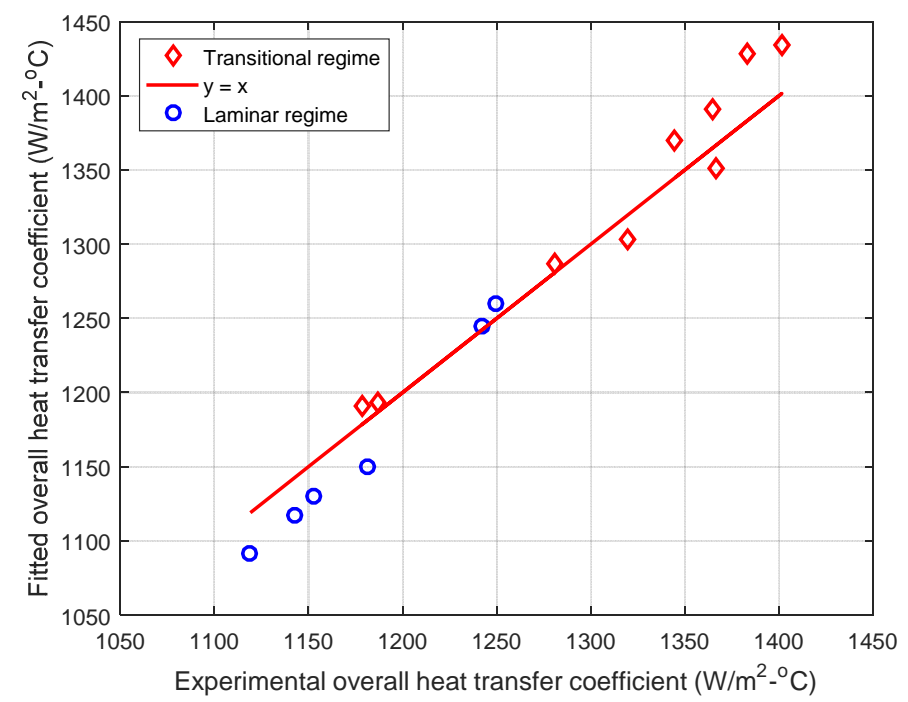

Fig. 11. Overall heat transfer coefficient comparison between the fitted model and experiments.

Finally, the fitted Nusselt number as a function of Reynolds number only is plotted in Fig. 12. It shows that the Nusselt numbers increased gradually with the increase of the Reynolds numbers, which was due to the helium velocity increase, resulting in a more turbulent fluid flow. The uncertainties of the Nusselt number correlations for the laminar and transition flow regimes were found to be $\pm 7 \%$ and $\pm 35 \%$, respectively, at 95\% confidence level. As mentioned, the behavior in the transition flow regime is complicated, resulting in the higher uncertainties. 


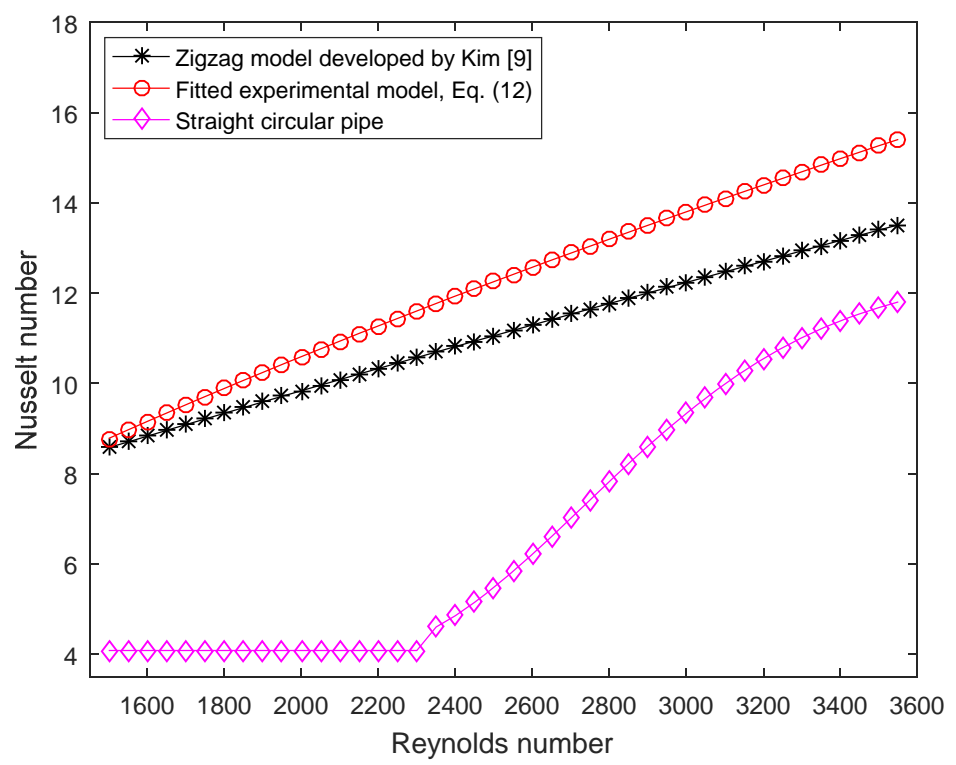

Fig. 12. Fitted Nusselt number correlation compared with the zigzag and straight circular pipe correlations.

Figure 12 also shows a comparison between the Nusselt number correlations shown in Eq. (12) and that gave in Kim’s correlation developed for zigzag flow channels [9]. There were some discrepancies between the two correlations, where Kim’s correlation had a slower increasing trend in the Nusselt number with the Reynolds numbers than the current correlations. As the Reynolds numbers increased, the differences between these two methods increased from 1 to $9 \%$. The disagreement can be attributed to the differences in the channel geometry. In addition, the boundary conditions for the top, bottom, and two side walls used in Kim's simulation were periodic [9], while the actual testing boundary conditions in the current experiments may not reflect the condition used in Kim's simulations.

A comparison of the Nusselt number correlation with fully-developed straight circular pipe correlations [19] is also presented in Fig. 12. The heat transfer coefficient in the current PCHE was about twice to three times that in straight circular pipes in the laminar flow regime and 1.5 to three times that in the transition flow regime. 


\section{Conclusion}

In this study, thermal-hydraulic performance of a reduced-scale zigzag-channel PCHE was investigated experimentally in a high-temperature helium test facility at The Ohio State University. The maximum PCHE test temperature reached $802^{\circ} \mathrm{C}$. It was found for both the Fanning friction factor and Nusselt number that the critical Reynolds number at laminar-to-turbulent flow transition was around 2,200 for zigzag channels. Pressure drop characteristics obtained from the experiments presented some discrepancies between the hot side and cold side, which could be mainly attributed to the manufacturing variations. The zigzag-channel Fanning friction factors were two to three times those in straight circular pipes in the laminar flow regime and one to two times greater than those in the transition flow regime. New pressure drop and heat transfer correlations for the zigzag channels with a roundness at each bend were developed based on the experimental data. Comparison of the available correlations with the proposed correlations showed that the available correlations under the predicted heat transfer performance of the current PCHE possibly due to geometrical differences in the flow channels. Compared to the results in straight circular pipes, PCHEs with zigzag channels have better overall heat transfer performance.

\section{Nomenclature}

a constant in the heat transfer correlation, Eq. (4)

$A_{c} \quad$ channel cross-sectional area

$A_{s} \quad$ heat transfer area

$c \quad$ constant in the heat transfer correlation, Eq. (4)

$d_{h} \quad$ channel hydraulic diameter

$f \quad$ Fanning friction factor 
G mass flux

$h \quad$ heat transfer coefficient

$i \quad$ inlet surface into the PCHE core

$K_{c} \quad$ contraction loss coefficient

$K_{e} \quad$ exit loss coefficient

$l \quad$ flow length

$\dot{m} \quad$ helium mass flow rate

n number of regression runs

$N \quad$ number of experimental data points

$\mathrm{Nu} \quad$ Nusselt number

$o \quad$ outlet surface of the PCHE core

$\Delta p \quad$ pressure drop

$\dot{Q} \quad$ heat conduction rate

$R_{w} \quad$ wall thermal resistance

Re Reynolds number

$S \quad$ residual

T temperature

$U \quad$ overall heat transfer coefficient

$x \quad$ zigzag channel inlet outlet surface

$\mathrm{X}$ an effective heat transfer region in the test PCHE

y $\quad$ zigzag channel outlet surface

$\mathrm{Y}$ an effective heat transfer region in the test PCHE

$\mathrm{Z}$ an effective heat transfer region in the test PCHE 


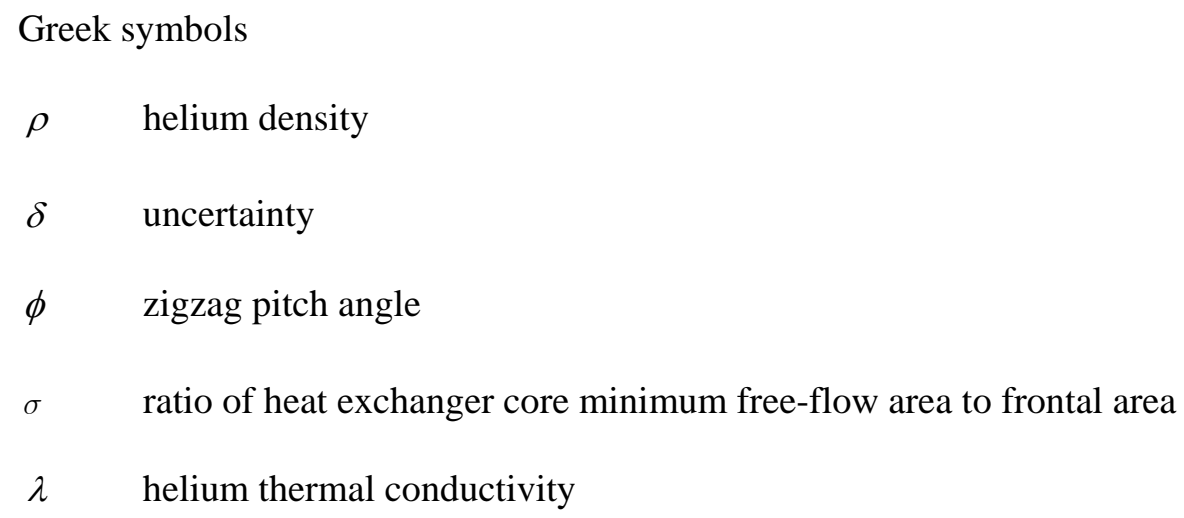

\section{Acknowledgements}

This research is being performed using funding received from the U.S. Department of Energy Office of Nuclear Energy's Nuclear Energy University Programs. The first author wish to thank Dr. Sai Mylavarapu for his work of constructing the high-temperature helium test facility. The assistance provided by Qiuping Lv and Kevin Wegman of The Ohio State University is also appreciated.

\section{References}

1. J. Nestell and S. Sam, “ASME Code Considerations for the Compact Heat Exchanger,” ORNL/TM2015/401, Oak Ridge National Laboratory, 2015. 
2. P. Sabharwall, E.S. Kim, M. McKellar, N. Anderson, and M. Patterson, "Process Heat Exchanger Options for the Advanced High Temperature Reactor,” INL/EXT-11-21584, Idaho National Laboratory, 2011.

3. S.K. Mylavarapu, "Design, Fabrication, Performance Testing, and Modeling of Diffusion Bonded Compact Heat Exchangers in a High-temperature Helium Test Facility,” Doctoral dissertation, The Ohio State University, 2011.

4. R.K. Shah and D.P. Sekulic, “Fundamentals of Heat Exchanger Design,” John Wiley \& Sons, 2003.

5. Heatric, http://www.heatric.com/typical characteristics.html, accessed on December 25, 2015.

6. K. Nikitin, Y. Kato, and L. Ngo, "Printed Circuit Heat Exchanger Thermal-hydraulic Performance in Supercritical $\mathrm{CO}_{2}$ Experimental Loop,” International Journal of Refrigeration, 29, pp. 807-814, 2006.

7. N. Tsuzuki, Y. Kato, and T. Ishiduka, “High Performance Printed Circuit Heat Exchanger,” Applied Thermal Engineering, 27, pp. 1702-1707, 2007.

8. T.L. Ngo, Y. Kato, K. Nikitin, and T. Ishizuka, "Heat Transfer and Pressure Drop Correlations of Microchannel Heat Exchanger with S-shaped and Zigzag Fins for Carbon Dioxide Cycles,” Experimental Thermal and Fluid Science, 32, pp. 560-570, 2007.

9. I.H. Kim, "Experimental and Numerical Investigations of Thermal-hydraulic Characteristics for the Design of a Printed Circuit Heat Exchanger (PCHE) in HTGRs,” Doctoral dissertation, Korea Advanced Institute of Science and Technology, 2012.

10. S.K. Mylavarapu, X. Sun, R.E. Glosup, R.N. Christensen, and M.W. Patterson, "Thermal Hydraulic Performance Testing of Printed Circuit Heat Exchangers in a High-temperature Helium Test Facility,” Applied Thermal Engineering, 65, pp. 605-614, 2014.

11. S.K. Mylavarapu, X. Sun, and R.N. Christensen, "Hydrodynamically Developing and FullyDeveloped Laminar Flows in a Semicircular Duct: Analytical and Computational Analyses,” Nuclear Science and Engineering, 182, pp. 319-331, 2016. 
12. N. Bartel, M. Chen, V.P. Utgikar, X. Sun, I.H. Kim, R.N. Christensen, and P. Sabharwall, “Comparative Analysis of Compact Heat Exchangers for Application as the Intermediate Heat Exchanger for Advanced Nuclear Reactors,” Annals of Nuclear Engineering, 81, pp. 143-149, 2015.

13. M. Chen, X. Sun, R.N. Christensen, I. Skavdahl, V.P. Utgikar, and P. Sabharwall, "Fabrication and Testing of a High-temperature Printed Circuit Heat Exchanger,” NURETH-16, Chicago, IL, August 30-September 4, 2015.

14. H.H. Khan, A.A. M, A. Sharma, A. Srivastava, and P. Chaudhun, “Thermal-hydraulic Characteristics and Performance of 3D Wavy Channel based Printed Circuit Heat Exchanger,” Applied Thermal Engineering, 87, pp. 519-528, 2015.

15. M. Chen, "Design, Fabrication, Testing, and Modeling of a High-temperature Printed Circuit Heat Exchanger,” Master thesis, The Ohio State University, 2015.

16. H.F. Khartabil and R.N. Christensen, “An Improved Scheme for Determining Heat Transfer Correlations from Heat Exchanger Regression Models with Three Unknowns,” Experimental Thermal and Fluid Science, 6, pp. 808-819, 1992.

17. F. Pra, P. Tochon, C. Mauget, J. Fokkens, and S. Willemsen, "Promising Designs of Compact Heat Exchangers for Modular HTRs Using the Brayton Cycle,” Nuclear Engineering and Design, 238, pp. 3160-3173, 2008.

18. D.E. Dim, M.H. Kim, J.E. Cha, and S.O. Kim, "Numerical Investigation on Thermal-hydraulic Performance of New Printed Circuit Heat Exchanger Model,” Nuclear Engineering and Design, 238, pp. 3269-3276, 2008.

19. J. Figley, X. Sun, S.K. Mylavarapu, and B. Hajek, "Numerical Study on Thermal Hydraulic Performance of a Printed Circuit Heat Exchanger,” Progress in Nuclear Energy, 68, pp. 89-96, 2013. 